\title{
Comunicación de la Ciencia desde la Mirada de los Investigadores Universitarios: entre el Indicador y la Vocación
}

\author{
Daniela Lazcano-Peña, Genny C. Viedma y Tomás V. Alcaino \\ Escuela de Periodismo, Pontificia Universidad Católica de Valparaíso, Avda. Universidad 330, Valparaíso -Chile. (e-mail: \\ daniela.lazcano@pucv.cl; genny.viedma@pucv.cl; tomas.alcaino.m@mail.pucv.cl)
}

Recibido Mar. 5, 2019; Aceptado May. 2, 2019; Versión final Jun. 10, 2019, Publicado Dic. 2019

\begin{abstract}
Resumen
Se explora y analiza las valoraciones de una muestra de investigadores/as chilenos/as sobre el quehacer de difusión y divulgación de los resultados de su investigación. Esto se hace a través del análisis de tres variables específicas: posicionamiento personal frente a la comunicación de la ciencia, obstáculos para la comunicación de la ciencia, y estrategias para potenciar la comunicación de la ciencia. La metodología de investigación combinó técnicas cualitativas y cuantitativas: entrevistas semiestructuradas a una muestra de 56 investigadores y encuesta online a una muestra de 110 investigadores. Los resultados indican que hay disparidad entre la difusión académica y la divulgación al medio externo del trabajo académico; mientras la difusión académica es parte de las exigencias e indicadores laborales, la divulgación al medio se enmarca en motivaciones de responsabilidad social de carácter personal. Se concluye que es necesario desarrollar una política institucional universitaria que equilibre los incentivos entre la difusión académica y las actividades de vinculación con el medio.
\end{abstract}

Palabras clave: comunicación de la ciencia; difusión de la ciencia; comunidad científica, rol social de la ciencia

\section{Science Communication from the Perspective of University Researchers: between the Indicator and the Vocation}

\begin{abstract}
The objective of this paper is to explore the valuation of a sample of Chilean researchers on their work in science communication. This is done through the analysis of three specific variables: personal positioning about communication of the science, obstacles to the communication of science, and strategies to improve the communication of science. The methodology combined qualitative and quantitative techniques: semistructured interviews to a sample of 56 researchers and online survey to a sample of 110 researchers. The results indicate that there is a disparity between academic diffusion and dissemination to the public; while academic diffusion is part of the demands and labor indicators, public dissemination is related to personal motivations inspired by social responsibility. It is concluded that it is necessary to develop a university institutional policy that balances the incentives between academic diffusion and activities related to public engagement.
\end{abstract}

Keywords: knowledge communication; dissemination of science; scientific community; social role of knowledge 


\section{INTRODUCCIÓN}

Si hablamos del trabajo que se realiza en universidades y centros de estudio, pensaremos en investigación: en la generación de nuevos conocimientos que entreguen nuevos elementos de análisis y reflexión, que mejoren un aspecto específico de la vida de una comunidad con avances teóricos o desarrollos tecnológicos. En definitiva, investigación con impacto social y sentido de servicio público, que aporte -desde cualquiera que sea su especialidad disciplinaria- a la construcción de una mejor sociedad. Consensuado ya el sentido social de la investigación y el desarrollo científico, y de la mano de este, vendrá una segunda necesidad: su "salida" de las aulas o laboratorios, para que los avances ahí generados no queden encerrados o ensimismados en la academia sino que sean conocidos y comprendidos por sus beneficiarios. En otras palabras, asumimos que parte de las tareas o funciones sociales de la investigación -sea cual sea su especialidad- es no solo aportar al desarrollo científico y tecnológico de la sociedad, sino también a su conocimiento, comprensión e información.

No es novedad que actualmente vivimos en una relación directa, constante y cotidiana -quizás a veces sin notarlo- con los resultados del trabajo científico, reflejado en productos, servicios o soluciones a problemas prácticos, lo que potencia el estrechamiento del vínculo entre la ciencia y el desarrollo de las sociedades contemporáneas. Vínculo que además despertaría nuestro interés informativo. Al menos, eso se puede desprender de los datos de la Encuesta Nacional de Percepción Social de la Ciencia y la Tecnología en Chile (Conicyt, 2016), que muestra que entre los niveles de interés por categorías temáticas como Deportes, Tecnología, Policial y Delictual, Ciencia, Cine y Teatro y Política, la Ciencia ocupa el cuarto lugar de las preferencias, resultando interesante para el $58,1 \%$ de la población sobre 15 años, y residente en Chile. Por otro lado, un $68,4 \%$ encuentra interesante a la Tecnología, posicionándola en el segundo lugar con mayor nivel de interés, y muy cercano al primer lugar que ocupa el interés por Deportes que alcanza un $68,8 \%$. Si bien el nivel de interés respecto a ambos aspectos-Ciencia y Tecnología- parece positivo, la situación no es la misma cuando se piden respuestas respecto del nivel de información que tienen sobre los mismos temas: Ciencia ocupa el primer lugar -con un 76,9\%- entre las personas que se sienten poco o nada informadas, mientras que la temática de Tecnología alcanza un 65,2\% en el mismo indicador. Es decir, los temas sobre ciencia y tecnología nos parecen interesantes, pero no contamos con suficiente información sobre ellos.

A partir de los resultados entregados por la Comisión Nacional de Ciencia y Tecnología a través de esta encuesta, nos interesamos por explorar sobre la tarea de comunicación de la ciencia y el conocimiento, y en específico sobre la valoración con que los propios generadores de este nuevo conocimiento, asumen esta tarea. Esto, sobre todo si asumimos que "acercar la ciencia a la sociedad es, en parte, responsabilidad de los científicos, y muy especialmente de aquellos que reciben fondos públicos para su investigación" (Lascurain y Sanz, 2009), y donde parte importante de esta responsabilidad se concentraría en las universidades, las que "deben promover mecanismos y acciones que faciliten el acceso de la opinión pública a la investigación y a la docencia que realizan" (Lascurain y Sanz, 2009). Entre estas acciones, hoy es posible identificar abordajes desde las unidades de comunicaciones y prensa existentes en muchas -si no en todas- las universidades, por ejemplo, pero también -como se observa en la literatura internacional- en una creciente preocupación por incorporar en la propia formación académica de los nuevos científicos de diversas áreas disciplinarias, competencias e interés sobre su rol de comunicadores de la ciencia (Brownell, Price y Steinman, 2013; Clarkson, Houghton, Chen y Rohde, 2018; Miller y Fahy, 2009; Silva y Bultitude, 2009; Stuart, 2013).

Actualmente, en Chile, las universidades juegan un rol sustancial en el desarrollo científico y tecnológico del país, pues muchas de ellas han sumado a su tarea de formación de capital humano -con su actividad docente de pre y postgrado- la investigación y vinculación con el medio como ejes esenciales de su misión institucional. De hecho, y según la VI Encuesta Nacional sobre Gasto y Personal en I+D 2016 (Ministerio de Economía, 2017) el sector Educación Superior fue el que más ejecutó gasto en investigación y desarrollo en el país, con 234.016 millones de pesos, lo que representa el 38,5\% del total del gasto nacional y considera que estas unidades son financiadas principalmente por el Estado. Un año después, en la séptima versión de la misma encuesta, el gasto en el sector había aumentado al 42\% (Ministerio de Economía, 2018). En el resumen ejecutivo de esta medición se expone que gran parte de los especialistas desarrollan sus investigaciones en el marco de actividades dentro de las instituciones de educación superior: entre investigadores, técnicos y personal de apoyo u otro personal, había en 201515.261 trabajadores de jornada completa dedicados a la investigación en nuestro país, de ellos 7.190 lo hacían desde las universidades, y en 2016 este número aumento a 8.061 (Ministerio de Economía, 2017). Los datos se condicen por los entregados en el Informe de Conicyt 2015-2018, que indica que la mayor cantidad de investigadores chilenos se concentra en el sector académico.

\section{MARCO TEÓRICO}

La unión de ambos elementos -la necesidad de promover la cultura científica y el rol de las universidades en la tarea de generación y socialización de nuevo conocimiento- son los ejes y motivación de este trabajo, el 
que busca aportar evidencia sobre la valoración y experiencia de los propios investigadores e investigadores universitarios en Chile frente a la tarea de comunicar la ciencia. Sobre este punto, y antes de continuar, creemos necesario realizar una aclaración: si bien hemos hablado de comunicación de la ciencia, la entendemos acotada no a las tradicionalmente denominadas ciencias duras, sino que incluimos todo tipo de conocimiento científico y académico generado en las diferentes áreas disciplinarias de las universidades. Esta mirada amplia, como se verá más adelante, se materializará en la configuración multidisciplinaria de la muestra.

Resulta casi evidente destacar cuál es el compromiso que enlaza a un establecimiento universitario con su realidad social, es decir, su deber ser hacia ésta mediante la elaboración de conocimiento científico, y su posterior incorporación al sistema social, generando de esta manera, un aporte para que mejore la calidad de vida de sus integrantes (Montenegro y Pujol, 2013). Sin embargo, y quizás en contradicción con este deber ser, es notoria la entrada del componente del mercado dentro de la ecuación educativa universitaria, el que se hace notar en la reestructuración de su mirada, en cuanto prioriza la satisfacción de demandas productivas mercantilizadas funcionales y especializadas (Guzmán, 2009). Así, la universidad muchas veces criticada como una torre de marfil en términos de producción de conocimiento, al estar supeditada a estándares económicos, terminó por incorporar dinámicas laborales desfavorables respecto a la actividad del docente investigador (Guzmán, 2009), prácticas que guardan relación con exigencias académicas al investigador para que este difunda su trabajo en formatos específicos, como, por ejemplo, papers y/o publicaciones en revistas indexadas en desmedro de otro tipo de vinculaciones y/o devoluciones para con la sociedad (Guzmán, 2009).

Así entonces, esta mirada de la universidad centrada en determinados indicadores abordaría la comunicación del conocimiento solo desde una arista acotada y destinada a la propia comunidad científica. Sobre este punto, es importante plantear la distinción entre dos conceptos vinculados a la comunicación de la ciencia, pero con connotaciones y alcances diferentes. Por una parte, la palabra difundir, que posee un estrecho vínculo con la tarea de los investigadores de dar a conocer su trabajo en un campo netamente académico, mediante la participación en charlas, congresos, publicaciones y otro tipo de acciones, por lo que se trata de un tipo de discurso que "contiene un conjunto de elementos o signos propios de un discurso especializado y una estructura que se constituyen en factores clave a la hora de su evaluación" (Espinosa, 2010). En este orden, se encuentran, por ejemplo, los artículos científicos publicados en revistas especializadas, papers y presentaciones en congresos, los que tienen una estructura, lenguaje y aspectos específicos que permiten la posterior evaluación de los resultados de las investigaciones científicas.

Por otra, divulgar, concepto que implica un plan de movimientos que posibiliten la circulación de los conocimientos científicos en ambientes populares a través de un gran espectro de vías: exposiciones en museos, conferencias, bibliotecas, revistas, cine, radio, televisión, prensa escrita, talleres escolares, colaboraciones y adaptaciones en teatro, y donde cada vez se van explorando las nuevas posibilidades y formatos que ofrecen las nuevas tecnologías (Büchi, 2017; Farinella, 2018). En este plano, la divulgación "es un instrumento para hacer equitativo el acceso a la cultura y al conocimiento. $Y$ debe cumplir el papel de presentar no solo los resultados de la investigación sino acercar los temas para que el ciudadano pueda entender lo que es importante y por qué" (Lascuraín y Sanz, 2009)

En esta línea, la universidad como entidad educativa ha cumplido un rol sustancial en la difusión y divulgación de conocimiento científico, en virtud de un proceso globalizador capitalista que necesita de la ciencia y tecnología para su reestructuración económica (Montenegro y Pujol, 2013). Teniendo esto presente, resulta interesante lo que puede aportar para la discusión la perspectiva del capitalismo cognitivo, que propone en grandes términos, que la economía genera una acumulación de bienes de riqueza, mediante la apropiación de los medios de producción que son informacionales, los que en última instancia son intangibles (Guzmán, 2009).

Este contexto facilita la creación de una nueva clase social denominada cognitariado, el que se caracteriza por la apropiación de estos medios de producción inmaterial, con la finalidad posterior de establecer relaciones de intercambio mercantil. Situación que los posiciona ventajosamente ante los empleados que solo pueden incorporarse al sistema capitalista mediante su fuerza de trabajo (Guzmán, 2009). Es bajo esta concepción que la universidad y el proceso de elaboración de conocimiento ha redefinido su práctica investigativaeducativa, en aras de una racionalidad de mercado que concluye por afectar estructuralmente a los profesionales dedicados a la academia, pues estos son pensados instrumentalmente para la generación de competencias individuales que el sistema económico globalizado instaura en la fuerza de trabajo contemporánea (Guzmán, 2009)

En este marco, la idea de calidad académica se encuentra ligada a indicadores cuantitativos de publicaciones en revistas académicas y su impacto con otras semejantes, en vez de aproximarse a la producción de conocimientos útiles $\mathrm{y} / \mathrm{o}$ al desarrollo de profesionales que propongan soluciones en función de las 
necesidades y demandas provenientes de los contextos sociales de los cuales las universidades son integrantes (Mato, 2013). Esta perspectiva teórica permite identificar y repensar no tan solo la práctica investigativa universitaria, sino que también replantear el rol del conocimiento científico en un contexto laboral que cada vez más se enfrenta a una institucionalización validadora, construida mayormente desde lógicas de mercado. Este escenario pone nuevamente de relieve, creemos, el que pueden cumplir las universidades para estrechar esta brecha, así como para el entendimiento de que su correcto desarrollo requiere de una actitud proactiva de especialistas que estén dispuestos a realizarla. Y es precisamente en las universidades, como ya hemos indicado, donde se encuentran gran parte de los investigadores del país.

\section{DISEÑO METODOLÓGICO}

Sentada ya la relevancia de la comunicación de la ciencia -en sus dimensiones de difusión y divulgacióncomo parte del propio trabajo de investigación, en este texto nos centramos en la voz de los propios investigadores e investigadoras. Pues nuestro interés es conocer cómo ellos experimentan y valoran sus actividades comunicación de la ciencia. ¿Cómo comunican su trabajo? ¿Qué los motiva a hacerlo? ¿Qué obstáculo o facilidades encuentran en esta tarea?

En este contexto, el objetivo de este trabajo es explorar las valoraciones de una muestra de investigadores/as chilenos/as sobre el quehacer de difusión y divulgación de los resultados de investigación. Para alcanzar este objetivo, nos centraremos en el análisis de tres variables específicas: (i) Posicionamiento personal frente a la comunicación de la ciencia; (ii) Obstáculos para la comunicación de la ciencia; y (iii) Estrategias para potenciar la comunicación de la ciencia. Para abordar estos objetivos se presenta los resultados de tres proyectos de investigación, autónomos pero vinculados. Por una parte, y como marco, el proyecto "Investigación en Comunicación en Chile: estado del arte y análisis de las características y proyecciones de la comunidad científica nacional" [COD Fondecyt 11160842], cuyo objetivo es caracterizar el campo académico de la Comunicación en Chile, considerando sus dimensiones científica-práctica de producción de conocimiento de los investigadores e investigadoras y educativa-reproducción de conocimiento a través de la docencia universitaria. Si bien el proyecto considera la comprensión del campo desde múltiples variables trayectorias académicas, institucionales y disciplinarias de los/as investigadores e investigadores; temáticas, objetos y problemas de investigación predominantes, enfoques metodológicos, entre otras variables- en este artículo en específico buscamos analizar su valoración sobre el impacto social de su investigación y su consiguiente proceso de comunicación; públicos y espacios en que comunica sus resultados de investigación; y las motivaciones, obstáculos o estrategias que enfrenta para la elección de esos espacios.

Estas dimensiones se profundizaron además, y en específico, en dos trabajos de graduación desarrollados al alero del Fondecyt, para pregrado y postgrado respectivamente: "Asimetrías en la producción de conocimiento: hacia una horizontalidad de los resultados de investigación en comunicación" y "Estado de la divulgación del conocimiento en las universidades del Consejo de Rectores de Valparaíso". A partir de estos trabajos de investigación, la valoración de las acciones de comunicación de la ciencia por parte de los propios investigadores/as se abordó desde técnicas cualitativas y cuantitativas. Desde la perspectiva cualitativa, se trabajó con dos muestras de investigadores/as. Por una parte, se realizaron entrevistas semiestructuradas a una muestra de 40 investigadores e investigadoras vinculados al campo de estudio de la Comunicación en Chile (22 hombres y 18 mujeres, de 14 universidades de todo el país), y seleccionados en el marco del proyecto Fondecyt a partir de su trayectoria académica en el área, y sus indicadores de productividad científica (proyectos, comunicaciones en congresos, y artículos académicos).

Estos resultados se ampliaron disciplinariamente con un segundo corpus de 16 entrevistas semiestructuradas a investigadores de universidades del Consejo de Rectores de Valparaíso (CRUV): Pontificia Universidad Católica de Valparaíso (PUCV), Universidad Técnica Federico Santa María (UTFSM), Universidad de Valparaíso (UV) y Universidad de Playa Ancha (UPLA). A modo de contexto, cabe indicar que el sistema de educación superior en Chile cuenta con una diversificación de tres tipos de instituciones: centros de formación técnica, institutos profesionales y universidades. Además, y más allá de su propiedad -pública o privada- sino que considerando su desarrollo histórico y rol público, pueden clasificarse como tradicionales y no tradicionales. Las universidades consideradas en esta muestra corresponden a la sección regional -CRUVdel Consejo de Rectores de Universidades Chilenas (CRUCH), grupo de 27 instituciones estatales y públicas no estatales, que cuentan con plena autonomía y cuyo objetivo principal es procurar la excelencia académica y el desarrollo de la investigación y la extensión universitaria.

Dada la amplitud y desconocimiento del universo -totalidad de académicos de las cuatro universidades del CRUV con actividades de investigación- para la conformación de la muestra de investigadores se trabajó en base al muestreo de bola de nieve, iniciando el proceso con informantes claves (representantes de las áreas de investigación de las casas de estudio en colaboración con el PAR Explora Valparaíso), quienes recomendaron el nombre de los primeros entrevistados en base al criterio de considerar a investigadores que 
destacaran en su proceso de comunicación de la ciencia en sus aristas de divulgación y difusión. Finalmente, se logró concretar una muestra con 16 entrevistas (9 a investigadores y 7 a investigadoras) compuesta por cinco en la Universidad de Valparaíso, cuatro de la Pontificia Universidad Católica de Valparaíso y en la Universidad Técnica Federico Santa María y tres en la Universidad de Playa Ancha. En cuanto a áreas del conocimiento, y al tratarse el muestreo de bola de nieve de un tipo de configuración no probabilística, se intencionó la diversidad disciplinaria, logrando representación de investigadores e investigadoras de Geografía, Química, Biología, Ingeniería y Medio Ambiente, Arquitectura, Física, Ingeniería Informática, Farmacia, Fonoaudiología, Estadística, Ingeniería Electrónica, Literatura y Matemática.

Desde la perspectiva cuantitativa, en tanto, se realizó una encuesta online con preguntas de respuestas cerradas también a investigadores que hubiesen realizado actividades de divulgación en las universidades del CRUV. En la encuesta se incluyeron preguntas para saber qué actividades de difusión y divulgación han realizado los encuestados, y para qué públicos, así como para reconocer si en sus universidades o unidades de trabajo existe una figura institucional que se encargue del desarrollo de esta área. Finalmente se dispuso una categoría de valorización de algunas afirmaciones configuradas con el fin de Conocer su percepción respecto a las motivaciones, formas y disposición personal como institucional para con la divulgación. En este caso se utilizó la plataforma digital Google Form, para la realización de cuestionarios, lo que permitió obtener de forma ordenada y analítica gráficos con resultados que ayudaron a contrastar la información general con la específica y más en profundidad que otorgaron las entrevistas. La muestra de la encuesta se generó gracias al envío a través del PAR Explora Valparaíso, en la que se encuentran 995 investigadores de distintas universidades de la Región y cuya tasa de apertura fue de un $43 \%$, es decir, sólo este porcentaje de los encuestados abrió el correo a través del que se les hizo llegar el cuestionario. De estas aperturas se obtuvo un total de 110 respuestas al cuestionario completo, por lo que forman la muestra final. Los investigadores que más respondieron son la Pontificia Universidad Católica de Valparaíso con un $56.5 \%$, seguidos de la Universidad de Valparaíso con un 17,6\%. Con porcentajes menores se encuentran la Universidad Técnica Federico Santa María y la Universidad de Playa Ancha. Respecto a las áreas de estudio desde las que provienen, la mayoría corresponde a las ciencias básicas e ingeniería, seguidos de áreas de comunicación y humanidades.

\section{RESULTADOS}

Para la organización de este apartado seguiremos la estructura de los objetivos específicos ya reseñados. En cada punto, además, se complementarán los resultados obtenidos en las entrevistas de la fase cualitativa con los datos derivados de la encuesta on line. La obtención de resultados de las entrevistas semiestructuradas se realizó a través de un análisis de contenido temático de cada una de las respuestas, basado en los principios de la teoría fundamentada (Glaser y Strauss, 1967), para identificar y categorizar las temáticas emergentes y sus significaciones o atributos asociados, a través de tres fases: (i) codificación abierta de temáticas emergentes; (ii) re-organización semántica por afinidad conceptual las temáticas emergentes; y (iii) re-codificación y configuración de un mapa de categorías (en este caso los objetivos o variables específicas a analizar), subcategorías vinculadas a la categoría principal, y significaciones o atributos que dotan de contenido, desde las propias citas de los entrevistados, a las respectivas subcategorías identificadas. Si bien en la fase cualitativa la conformación total de las entrevistas revisadas incluye variedad disciplinaria y de género, los datos no evidencian diferencias significativas entre estas variables. Por este motivo, la presentación de los datos se realizará de manera agregada, sin distinción de variables específicas.

Entre los entrevistados la respuesta es unánime: el trabajo de investigación debe tener, siempre, un sentido o impacto social. Es decir, se realiza investigación para beneficiar o mejorar una situación, para avanzar en una mejor comprensión teórica de un determinado fenómeno, o aportar de manera práctica y directa a resolver problemas o generar productos/servicios que contribuyan al mejor desarrollo de la vida de personas desde diversas áreas del conocimiento. De la mano de esta comprensión basal del sentido del trabajo científico, se desprenden subcategorías asociadas a este objetivo: las motivaciones que declaran los investigadores para realizar acciones de comunicación de su quehacer académico; y los ámbitos (o públicos) en que observan la expresión del impacto social de su investigación, y que redundará en determinados espacios y acciones de comunicación de la ciencia.

\section{Investigación con impacto social y la obligación de la comunicación}

Así como la investigación que se desarrolla tiene un rol público en beneficio de la sociedad, así también se comprende como una necesidad y responsabilidad, el compartir y comunicar sus resultados. Sobre este punto los resultados de la encuesta reflejan que el $90 \%$ de los encuestados está de acuerdo o muy de acuerdo con las afirmaciones que indican que sí están dispuestos a realizar tanto acciones de difusión como de divulgación del conocimiento, lo que refleja un interés significativo. 
Tabla 1: Mapa de resultados análisis de contenido temático

\begin{tabular}{|c|c|c|}
\hline Variables o categorías & Subcategorías & Atributos o significaciones \\
\hline \multirow{6}{*}{$\begin{array}{l}\text { Posicionamiento personal } \\
\text { frente a la comunicación de } \\
\text { la ciencia }\end{array}$} & \multirow{3}{*}{$\begin{array}{l}\text { Motivaciones para la comunicación } \\
\text { de la ciencia }\end{array}$} & Diálogo académico \\
\hline & & Devolución de apoyos públicos \\
\hline & & Fomento de cultura científica \\
\hline & \multirow{3}{*}{$\begin{array}{l}\text { Ámbitos de impacto social } \\
\text { del conocimiento }\end{array}$} & Aporte al desarrollo del país \\
\hline & & Aporte a la disciplina \\
\hline & & Aporte a la sociedad en general \\
\hline \multirow{8}{*}{$\begin{array}{l}\text { Obstáculos para la } \\
\text { comunicación de la ciencia }\end{array}$} & \multirow[t]{3}{*}{ Obstáculos para difusión } & Carga laboral de gestión \\
\hline & & Barrera idiomática \\
\hline & & Financiamiento \\
\hline & \multirow[t]{5}{*}{ Obstáculos para divulgación } & Indicadores académicos \\
\hline & & Financiamiento \\
\hline & & Habilidades comunicativas \\
\hline & & Noticiabilidad de la investigación \\
\hline & & Falta de innovación \\
\hline \multirow{5}{*}{$\begin{array}{l}\text { Estrategias para potenciar la } \\
\text { comunicación de la ciencia }\end{array}$} & \multirow[t]{2}{*}{ Estrategias para potenciar difusión } & Indexación y ranking \\
\hline & & Públicos y formatos de interés \\
\hline & \multirow{3}{*}{$\begin{array}{l}\text { Estrategias para potenciar } \\
\text { divulgación }\end{array}$} & Asociatividad \\
\hline & & Capacitación en comunicación \\
\hline & & Cambio en políticas institucionales \\
\hline
\end{tabular}

La tarea de comunicar se desarrollaría por tres motivaciones o razones principales. Por una parte, se comprende como parte del diálogo académico, es decir, aportar y comunicar en el espacio de la propia disciplina (lo que se relacionaría con la actividad de difusión, de acuerdo a lo que hemos establecido en el marco teórico), pero también se evidencian motivaciones divulgativas enmarcadas en la idea de aportar a la generación de una cultura científica en la sociedad en general, es decir, contribuir a la alfabetización ciudadana en materias de desarrollo científico, para que entiendan este conocimiento como un aspecto que está presente en prácticamente todo lo que se hace a diario, en las cosas más simples y que, por tanto, puede ayudar a mejorar la calidad de vida de las personas ["En mi caso la motivación de fondo creo que tiene que ver con la forma en que uno ve la ciencia, porque yo no veo el conocimiento como un medio para el desarrollo económico o científico netamente, sino que también lo veo como un medio para el desarrollo humano y social, entonces creo que es un derecho de las personas, así como saber leer o escribir, saber y apropiarse de un conocimiento" (Investigador, Pedagogía en matemáticas)].

Por otro lado, se repite una necesidad de "devolver" de una forma práctica lo que la sociedad, a través del Estado les ha entregado (devolución de apoyos públicos), haciendo referencia a los recursos que han recibido para el financiamiento de sus especializaciones, formación como magísteres o doctores y para el desarrollo de sus investigaciones, lo que los motiva a comunicar aquellos resultados relevantes al público general, entendiendo que esos recursos estatales forman parte de lo que cada ciudadano aporta con sus impuestos, por ejemplo ["Yo fui becado por el Estado a través de una beca Conicyt para realizar mi doctorado, y gran parte de mi desarrollo profesional se lo debo a fondos públicos que salieron del bolsillo de la gente. Hasta cierto punto es, entonces, una responsabilidad de devolver eso a la sociedad. Nunca he cobrado por una charla y he hecho cursos gratis a ONGs sin fines de lucro, esa es una motivación" (Investigador, Química)].

Si bien en el marco teórico hemos señalado la diferencia, dentro del campo de la comunicación de la ciencia, entre difusión y divulgación, esta distinción no está presente en términos conceptuales entre los entrevistados, por lo que en el modo de comprender sus acciones de comunicación científica, se destacan tanto las acciones hacia la propia comunidad académica de pares (difusión) y hacia la comunidad (divulgación). Tomando ambas aristas, y a partir de las respuestas de los mismos entrevistados, es posible evidenciar los ámbitos en 
que los investigadores observan que se materializa el rol público de su trabajo y, por ende, la comunicación de este: desarrollo del país; la propia disciplina y la sociedad en general.

En esta subcategoría además, y como se observa en la siguiente tabla, es posible observar vínculos entre estos ámbitos y los públicos que identifican como receptores de la comunicación de sus resultados o, desde otra perspectiva, beneficiarios del impacto social de sus investigaciones, y los espacios de difusión/divulgación en que se operacionalizan sus acciones de comunicación. En este vínculo es posible evidenciar también una relación entre la percepción de frecuencia de realización de una acción de comunicación, versus la percepción de su impacto.

Tabla 2: relación ámbitos y acciones de impacto social de la investigación

\begin{tabular}{|c|c|c|c|c|}
\hline Ámbitos & Públicos & Acciones & $\begin{array}{l}\text { Percepción de } \\
\text { frecuencia }\end{array}$ & $\begin{array}{l}\text { Percepción de } \\
\text { impacto }\end{array}$ \\
\hline $\begin{array}{l}\text { Desarrollo del } \\
\text { país }\end{array}$ & Política pública & $\begin{array}{l}\text { Mesas y comités de } \\
\text { expertos }\end{array}$ & Baja & Alta \\
\hline \multirow[t]{4}{*}{ Disciplina } & \multirow{2}{*}{$\begin{array}{l}\text { Comunidad académica } \\
\text { (pares) }\end{array}$} & Revistas académicas & Alta & Alta \\
\hline & & Congresos & Alta & Baja \\
\hline & \multirow{2}{*}{$\begin{array}{l}\text { Comunidad académica } \\
\text { (estudiantes) }\end{array}$} & Libros & Baja & Alta \\
\hline & & Seminarios & Alta & Baja \\
\hline \multirow{4}{*}{$\begin{array}{l}\text { Sociedad en } \\
\text { general }\end{array}$} & \multirow{3}{*}{$\begin{array}{l}\text { Comunidad escolar } \\
- \\
\text { Organizaciones de la } \\
\text { sociedad civil }\end{array}$} & Talleres y charlas & Baja & Alta \\
\hline & & Libros (digitales) & Baja & Alta \\
\hline & & Redes sociales & Baja & Alta \\
\hline & Público general & Medios de prensa & Baja & Baja \\
\hline
\end{tabular}

Así, por ejemplo, para los investigadores, uno de los principales focos que debieran orientar el impacto social de la investigación es el aporte a políticas públicas, el que se materializa a través de la participación en mesas de expertos o comités de asesores. Sin embargo, si bien esta es una actividad a la que se da gran valor, se observa como una tarea a fortalecer, es decir, la participación en espacio de impacto de la política pública es menor de la que debiera desarrollarse ["En las políticas públicas del país, creo que ahí estamos en un debe, no puede ser que nosotros investiguemos, vamos a congresos, nos felicitamos entre nosotros y quedemos tan contentos, yo no quedo contenta" (Investigadora, Comunicación)]

En todas las entrevistas analizadas, y en la encuesta, se evidencia que un espacio de comunicación predominante es la difusión académica a través de publicación en revistas especializadas y participación en congresos y seminarios académicos. De hecho, el $73 \%$ declara que en el último año ha realizado actividades ligadas a la difusión entre pares, versus un $61 \%$ que ha realizado actividades dirigidas a público general (en especial charlas). Entre estos espacios son, sin duda, las revistas el espacio privilegiado tanto por su frecuencia como en su valoración dentro de las acciones de comunicación: es decir, se publica ampliamente y se considera un espacio apropiado para la difusión de resultados. Sin embargo, no sucede lo mismo con los congresos académicos, los que si bien cuentan con una alta frecuencia de realización, no siempre se valoran como espacios de difusión de calidad. Con excepción de determinados congresos de conocido reconocimiento en las diferentes áreas de especialidad, la sensación en términos generales es de una baja calidad e interés académico ["Uno va a conferencias, va a congresos, ve a la misma gente, conversa de los mismos temas... A mí cada vez me agotan un poco más las conferencias, más allá del turismo académico que uno hace y que todos hacemos, las conferencias dejan bastante poco, por la lógica de funcionamiento que tienen: conversaciones muy restringidas de 15 minutos, muchos paneles -porque hay que financiar el congreso- y poca instancia de trabajo" (Investigador, Periodismo)]

Otro ámbito de interés como parte de las acciones de comunicación son los propios estudiantes. De hecho, para algunos investigadores se trataría del foco esencial de la investigación: perfeccionar la propia docencia para la formación de mejores profesionales ["La institución académica, bajo las prerrogativas del modelo neoliberal en el que se están organizando en los últimos diez años, entiende la extensión como impacto y entiende el impacto como algún tipo de transferencia (...) pero el primer impacto que la universidad tiene sobre la sociedad son sus egresados, o sea, la docencia es la primera forma en que la universidad le entrega algo a la sociedad porque le entrega profesionales y, por lo tanto, en la formación docente debería haber un primer sello o una primera preocupación" (Investigador, Comunicación)]. Ese énfasis formativo se evidencia 
en otro de los públicos destacados como focos de comunicación de la ciencia: la comunidad escolar, espacio que se valora por su potencial de motivación de futuras generaciones, tanto si se realizan actividades directas con estudiantes (a través de instancias de PAR Explora, como Mil científicos mil aulas, por ejemplo, donde un $28 \%$ de los participantes de la encuesta dice haber participado el último año) o con docentes del sistema educativo.

Finalmente, otro de los focos de la comunicación de la ciencia sería la propia sociedad, ya sea en general (con actividades masivas y mediadas de divulgación) o más específicas destinadas en concreto a las organizaciones o actores sociales vinculados con las mismas investigaciones. En ese sentido, sucede una valoración análoga a lo observado en el ámbito de las políticas públicas: se valoran como un espacio necesario, pero al que se dedican pocas acciones específicas ["Uno lo hace de buena voluntad y siento que es parte de mi compromiso profesional el devolver la experiencia que uno va ganando con el tiempo. Trabajar con dirigentes sociales o vecinales tiene otro sentido, porque generalmente se trata de resolver problemas que los están afectando" (Investigadora, Ingeniería y medio ambiente); "Lo valoro mucho y considero que es bajo. En ciertos proyectos en que nosotros hemos trabajado, por ejemplo, con el proyecto de La Pintana sobre los niños reporteros (...) ahí la devolución fue en terreno porque nosotros estábamos permanentemente con ellos" (Investigadora, Comunicación)].

En cuanto a las apariciones en prensa, muchas veces impulsadas por los departamentos de comunicación de las propias universidades, y si bien tienen una presencia considerable en los datos de la encuesta (un $45 \%$ dice haber tenido apariciones en medios de comunicación en el último año), no son bien valoradas cualitativamente, en especial porque se considera como escaso el interés de los propios medios para el tratamiento de estos contenidos, sobre todos en los espacios no especializados ["De repente te invitan a una entrevista en la televisión, y cuando te están entrevistando el tipo está leyendo otras cosas, y esperando para pasar al deporte con el último gol de Alexis Sánchez. O sea, lo que tú dijiste no importa nada" (Investigador, Comunicación)].

\section{Obstáculos para la comunicación de la ciencia}

No hay sorpresa en la evidencia: los principales obstáculos en la comunicación científica se concentran en las acciones de divulgación. Sin embargo, y a pesar de esta concentración, sí aparecen también dificultades en la instancia de difusión entre la comunidad académica. Por una parte, y ante la exigencia de los indicadores institucionales (que desarrollaremos más adelante) que priorizan como parte del quehacer académico la redacción y publicación de artículos en revistas científicas, destaca como obstáculo el tiempo que se puede dedicar a esta tarea, en especial cuando el trabajo académico además de la docencia y la investigación se debe compatibilizar con carga laboral dedicada a la gestión universitaria ["La verdad es que mi difusión ha sido dentro de lo posible. A veces un artículo por año, a veces dos, pero no es una carrera prolífica en ese ámbito. Estoy un poco "al debe" porque yo en paralelo a todo esto, he estado con una carga administrativa super fuerte" (Investigador, Comunicación)] lo que afecta negativamente la "productividad académica" de los investigadores.

Vinculada a la publicación en revistas científicas, otra de las dificultades observadas -en especial entre los investigadores de ciencias sociales, humanidades y comunicación- es la escasez de revistas indexadas de corriente principal editadas en español, lo que devela la barrera idiomática, en especial por la exigencia del idioma nativo inglés de gran parte de estas publicaciones ["Apuntar a las revistas en inglés, que es una barrera doble porque, me ha pasado más de una vez, por mucho que hemos mandado a traducir a traductores profesionales, nos han llegado comentarios de revistas gringas sobre que igual es evidente que no es nativo" (Investigadora, Comunicación)]. El último obstáculo observado en esta subcategoría, es el financiamiento: ya sea para el pago de traducciones profesionales, por la exigencia recién reseñada, como por los altos costos de los congresos internacionales de reconocida calidad académica ["Los congresos no los elijo, veo lo que está disponible, digamos. Para eso he encontrado poco financiamiento, para participar, por ejemplo, en congresos internacionales. Entonces, lógicamente, participo más de los congresos nacionales y después congresos que sean más cercanos geográficamente" (Investigador, Comunicación)].

El financiamiento es también un obstáculo para la arista divulgativa de la comunicación científica. Los resultados de la encuesta arrojan que de los 110 cuestionarios respondidos, 67 dicen estar de acuerdo o totalmente de acuerdo con la afirmación que indica que no cuentan con recursos suficientes para el desarrollo de actividades de divulgación ["Cuando voy a charlas de colegio y otras actividades de este orden, es con mis propios recursos, es por amor al arte" (Investigadora, Química)]. Sólo en el caso de los entrevistados relacionados a áreas como literatura, pedagogía y salud, existe una sensación de que los recursos sí están a disposición, pero hay que saber dónde buscarlos, ya sea a través de fondos de las universidades o de gestión propia ["Yo creo que recursos hay, creo que es más difícil conseguir fondos de investigación que fondos para divulgación del conocimiento. (...) No creo que sea difícil hay que motivarse y saber con quién hablar y hacerlo en equipo" (Investigador, Literatura)] 
Sin embargo, y como ya hemos insinuado, el principal obstáculo para las acciones de divulgación se concentra - de acuerdo a las respuestas de los investigadores- en los indicadores del trabajo científico que valoran las universidades, y que se materializa en incentivos financieros $\mathrm{y} / \mathrm{o}$ en reconocimiento para el avance en la carrera académica de los investigadores. Y carrera en toda su amplitud semántica: pues es la publicación de artículos en revistas especializadas indexadas de corriente principal el factor principal que ubicará a los investigadores en mejores posiciones para promociones en jerarquía académica ["Como los profesores están obligados a buscar los puntos que les hacen su carrera académica en otros lados, van dejando de lado lo que no da puntos. Esto provoca un enredo que obliga a los profesores a estar un lado de publicaciones que lee muy poca gente entre sus mismos pares. Esa brecha no está resuelta, entonces, el académico que tiene ganas de hacerlo lo hace por motivo propio" (Investigador, Arquitectura)].

Como se desprende de la cita anterior, en la contracara, entonces, los investigadores de la muestra identifican que la realización de actividades de divulgación no otorga "puntos" o no son validadas para el crecimiento de su carrera. A pesar que de manera específica se pueden observar sistemas de incentivos o reconocimientos internos en las propias escuelas o institutos, se valora como carencia la ausencia de políticas institucionales que garanticen la valoración de las actividades de divulgación, más allá del discurso público [“En el discurso siempre dicen que es súper importante y que está en el plan estratégico, pero en la práctica tiene poco peso en la valoración, que significa que todos los profesores que están jerarquizados tienen un componente de variable del sueldo y todas las actividades de divulgación suman muy poco" (Investigador, Biología); "El tema de que esto no te ayude con la carrera académica influye, porque no hay incentivos de este orden, pero de todos modos algo se está avanzado en este tema al poner en los proyectos que es una responsabilidad entregar este conocimiento. No hay un incentivo como sí lo hay para la investigación, deberían considerar docencia, investigación y vinculación con el medio" (Investigadora, Ingeniería Informática)].

Aunque con menor frecuencia que el punto anterior, otro obstáculo que se identifica en el desarrollo de actividades de divulgación, son las habilidades comunicativas, o más bien la falta de competencias para poder expresar y adaptar las presentación de resultados u otros temas de interés de la investigación a diferentes públicos externos a la propia especialización disciplinaria ["Para que te logres hacer entender por un público como los niños tienes que subir un nivel en el esfuerzo que realizas para comunicarte, al revés de lo que se pensaría. Alto nivel tiene un profesor tal cual se le entiende todo y es capaz de hacerse entender por distintas audiencias" (Investigador, Arquitectura)]. Esta percepción del análisis cualitativo se condice con los datos de la encuesta, donde un total de 62 personas (56\% de la muestra) están de acuerdo o totalmente de acuerdo con la afirmación sobre la falta de herramientas comunicacionales para la divulgación.

Sobre este punto resulta interesante que si bien muchas universidades -quizás todas- cuentan con departamentos de comunicación para facilitar las actividades de divulgación de sus académicos, sobre todo las relacionadas con aparición en prensa, este apoyo pareciera aún insuficiente, o al menos los investigadores de la muestra no lo observan como un claro reemplazo de su percepción personal de falta de habilidades comunicativas ["No todos tienen las habilidades comunicacionales para llevar a cabo tareas de divulgación o un mensaje adecuado. En este sentido el que tiene que llevar bien los mensajes es el periodista, pero para eso uno tiene que entregar bien la información y revisar, para poder confiar en el otro. Es decir, es importante el trabajo multidisciplinario" (Investigadora, Ingeniería Informática)]

También vinculada a la acción de divulgación en prensa, un obstáculo es la noticiabilidad de los temas de investigación en general o sus resultados en particular. Estableciendo como noticiabilidad el interés o posibilidad de presentar como "noticia" algún tema o acontecimientos, observamos que los investigadores tienen la percepción de que sus temas de investigación son difíciles de mostrar en los medios, ya sea por su abstracción y poca aplicabilidad cotidiana o -y en especial para los investigadores de las áreas de periodismo y comunicación- por el escaso interés de los propios medios ["En nuestras áreas, ¿cuáles serían como los impactos al respecto? Bueno, son las participaciones en políticas públicas, la elaboración de observatorios, entre otros. Idealmente, el impacto principal debería estar en el ámbito de las comunicaciones, de los medios y de las telecomunicaciones, pero ahí no nos pescan" (Investigador, Comunicación)].

Finalmente, otro de los obstáculos que se desprende del análisis de las entrevistas es la falta de innovación con que los propios investigadores desarrollan las actividades de divulgación. Un ejemplo concreto: las charlas o seminarios abiertos a la comunidad, pero donde finalmente asisten solo estudiantes de los propios investigadores, no cumpliendo su finalidad divulgativa, sino redundando en la difusión entre la comunidad especializada o en proceso de especialización ["Quiero probar cosas distintas, porque lo típico que proponen los proyectos son páginas web, ¿cuántas miles de miles de páginas web existen, cómo las alimentas y cómo haces que funcionen? No me parece una buena opción, porque se lanza, queda ahí y nadie sabe que está ahí. O el tema de los seminarios, que también son formatos muy académicos" (Investigadora, Comunicación]. En este sentido, sí se observa una diferencia entre disciplinas, y una mayor predisposición hacia la innovación 
entre los investigadores de las áreas de ciencias "más duras", pues no son pocos los entrevistados que de manera autodidacta han comenzado a realizar sus propios canales de youtube, para apuntar a una mayor masividad de los contenidos científicos.

\section{Estrategias para potenciar la comunicación de la ciencia}

En la última parte del análisis sistematizamos las respuestas sobre las estrategias para potenciar la comunicación científica. Al igual que en el apartado anterior, separemos los resultados entre las dimensiones de difusión y divulgación. Más que potenciar, mejorar o incrementar la circulación de la investigación en los espacios de y para la propia comunidad científica (difusión) que, como hemos visto, es la arista más consolidada en cuanto a la dedicación de los propios investigadores como a su valoración e incentivo por parte de las instituciones de educación superior, sí se pueden observar algunos criterios que guían las acciones de difusión de los investigadores.

Entre estos criterios, el más marcado: la indexación de las revistas académicas. Si las universidades explícitamente valoran y reconocen esos espacios, es ahí donde los investigadores saben que deben enfocar su trabajo de difusión, canalizando sus tiempos y esfuerzos que, como vimos en el punto anterior, escasean entre la múltiple carga de tareas que debe asumir un profesor y profesora universitario ["Al principio yo estaba muy interesada en mostrar mi trabajo en las revistas que a mí me gustaba leer. Pero después dejé de hacer eso, y tengo que mostrarlo en las revistas que están indexadas y responder a todas esas presiones de productividad que hay en mi universidad, y en todas las universidades en general" (Investigadora, Comunicación)].

Criterio similar opera en la selección de congresos. Al respecto ya hemos revisado dos variables que nos permiten contextualizar y comprender estos resultados: las dudas sobre la calidad académica de algunos de estos espacios y, en contrapartida, el alto costo de los que sí cuentan con reconocimiento en las respectivas disciplinas. En ese contexto, entonces, la selección de los congresos estará marcada por su "ranking" o valoración dentro del respectivo campo de estudio o, como otra variable, la comunidad académica a la que congrega ["Trato de ir a las mismas conferencias, pero cada vez es más difícil también, porque los porcentajes de aceptación son de 30\%. Son el top del top" (Investigadora, Comunicación organizacional); "En conferencias, aparte de la de InCom -Congreso anual de la Asociación Chilena de Investigadores en Comunicación-, a la de la International Communication Asociation voy harto, pero si te soy sincera ahí es donde me encuentro con mis colegas gringos y porque me gusta seguir en contacto con ese mundo" (Investigadora, Comunicación)].

Esta mirada nos acerca al segundo atributo en la categorización las estrategias de difusión: los públicos y formatos de interés. Este atributo resulta interesante además, pues se plantea como contrapunto crítico a las exigencias y sobrevaloración por las revistas indexadas ["Las revistas quieren ser indexadas, y si son indexadas no son un espacio que necesariamente sea para la academia local. O sea, ¿cuál es la conversación que en términos locales estamos nosotros teniendo respecto de nuestros problemas en comunicación? Porque si yo publico en la revista asiática de estudios de comunicación, por ejemplo, que puede tener un alto impacto, no la puedo usar en clase, no la pueden leer mis estudiantes, es como ya... ¿y?" (Investigadora, Comunicación)].

En cuanto a la arista de divulgación, algunas estrategias planteadas apuntan a la asociatividad entre investigadores -lo que podría aportar a superar el obstáculo de la falta de recursos y asegurar un mayor alcance de convocatoria-; y al desarrollo de espacios de capacitación sobre habilidades comunicacionales en el propio proceso formativo de los futuros investigadores, es decir, la incorporación de asignaturas o al menos talleres dentro de las mallas curriculares de pregrado, que incentiven a los estudiantes desde su formación a realizar investigación con una mirada divulgativa y que les permita desarrollar habilidades blandas y comunicacionales que aporten en sus carreras profesionales, de manera que sean capaces de expresar lo que quieren lograr con sus investigaciones de manera comprensible ["Esto no sólo es importante, sino que también es factible y solucionaría un tema que tengo entendido que ya se está resolviendo un poco con los ramos de formación fundamental o los optativos. Éstos deberían responder a algunos ejes donde uno pudiera escoger un eje de habilidades blandas y comunicativas, lo que tendría lógica porque apuntaría al problema de la deficiencia de divulgadores de la ciencia" (Investigador, Química]. El entrenamiento en áreas como la redacción (científica y para público general), el uso de nuevas tecnologías como redes sociales y medios digitales, las técnicas de persuasión, les permitiría entrenarse al campo laboral y contar con herramientas para el desarrollo de su vida profesional, útiles para ellos, sería una forma indirecta de aportar a la cultura científica nacional, al hacerlos conscientes de la importancia de la comunicación de sus logros.

Pero finalmente, la estrategia más a largo plazo y marco de todas las posibles acciones para potenciar la divulgación sería, sin duda, un cambio en las políticas institucionales. Los entrevistados responden que sería 
muy provechoso y necesario que, así como existen fondos y políticas públicas y universitarias para la difusión de la investigación, también lo hubiese más para la divulgación, puesto que ambas son partes fundamentales del trabajo en investigación. Entre estas políticas derivadas de la gestión institucional, se presentan algunos aspectos importantes que justamente coinciden con los que se han mencionado anteriormente como los principales obstáculos que se generan a la hora de realizar la divulgación. De esta forma, la liberación de la carga horaria, la disponibilidad de recursos financieros, la facilitación de espacios, y la posibilidad de una generación de incentivos para la carrera académica derivada de las actividades de divulgación, son algunas de las formas que los investigadores ven como potenciales motores para que otros estén dispuestos a realizarla, así como para que ellos mismos puedan hacerlo de la mejor forma posible. Por otro lado, en este aspecto también se retoma la estrecha relación que debiesen tener las áreas de vinculación con el medio y los equipos de comunicaciones, con las facultades o departamentos disciplinarios, tanto para colaborar en las labores de gestión de las actividades de vinculación, como para desarrollar programas de talleres, asesorías o cursos especializados que otorguen herramientas y potencien las habilidades comunicacionales de los investigadores.

\section{DISCUSIÓN, ANÁLISIS Y REFLEXIONES}

El desarrollo de la comunicación de la ciencia avanza en su comprensión y reflexión como área de estudio, con literatura especializada que aborda desde su recorrido histórico en contextos específicos (Pérez y Olvera, 2017) hasta revisiones de alcances conceptuales y críticos (Guenther y Joubert, 2017; Kahan, 2015; Lamberts, 2017). En este contexto macro como marco reconocemos, por una parte, los límites de la investigación aquí presentada, pero también valoramos su aporte, pues en el entendido que ahondar en el universo de percepciones, experiencias y opiniones de la totalidad de investigadores e investigadoras de las universidades chilenas, sería inabordable, creemos que los resultados desarrollados, si bien corresponden a una muestra acotada, permiten aportar evidencia a varios supuestos que existen respecto al trabajo de comunicación del conocimiento científico que se realiza desde las universidades.

En este sentido, por ejemplo, constatamos la disparidad entre la difusión y la divulgación: la difusión se exige, se comprende como parte de las exigencias laborales. Es un indicador. La divulgación, en tanto, en muchos casos es vocación. Y es que es claro que esta nace de una motivación de carácter personal e incluso altruista de fomentar la cultura científica entre las personas que no tienen acceso a ella y que esto se transforma en un puente de beneficios y desarrollo social, pero que además, es una respuesta a la responsabilidad que conlleva el hecho de haber estudiado y realizado investigación gracias a fondos públicos, lo que genera una necesidad de devolución a modo de agradecimiento convertido en obra desde la comunicación de sus hallazgos y alcances científicos a los demás. Esto, a pesar de que en la actualidad no hay grandes incentivos de tiempo, recursos y de aspiraciones académicas que lo avalen.

De ahí las recomendaciones que los mismos académicos indican: abordar la política institucional, de manera que los incentivos y acciones de vinculación con el medio e investigación vayan de la mano con los lineamientos planteados en sus misiones y declaraciones de deber ser para con la sociedad. En este sentido destacan que, en los últimos años, se ha trabajado en un marco de mejores condiciones y en pro de una importante intención del desarrollo para ésta área, pero que aún se trata de lineamientos que deben ir posicionándose entre autoridades y los mismos pares académicos. Pasos en caminos similares sí se están dando en otras partes del mundo. A principios de este mes (diciembre 2018), el Crue Universidades Españolas y la Fundación Española para la Ciencia y la Tecnología (FECYT) lanzaron la Guía de Valoración de la Actividad de Divulgación Científica del Personal Académico e Investigador, lo que permitirá avanzar en la institucionalización de indicadores que permitan valorar el trabajo divulgativo de la comunidad científica española. ¿Un horizonte posible para nuestro país? Por qué no.

Creemos necesario apuntar que el objetivo del artículo no ha sido realizar una apología del proceso de divulgación de la ciencia que desemboque en una exigencia más dentro de los marcos académicos profesionales al momento de investigar sino, aportar documentación con la finalidad de identificar problemáticas en instancias de divulgación y repensar los roles sociales del conocimiento científico. ¿Y por qué lo hacemos desde el campo de la Comunicación -donde nos ubicamos disciplinariamente-? En primera instancia y como es evidente, tanto la difusión como la divulgación, son actividades de extensión comunicacional, donde contar con ciertas lógicas y procesos de trabajo conjunto e interdisciplinar, podría aportar para desarrollarlas de la mejor forma posible.

En este sentido es que se transforma en un desafío que puede ser cubierto desde el campo de las comunicaciones y puede, incluso, abrir un puente entre la relación científico/periodista, que no siempre es fluida, sobretodo en el desarrollo del trabajo de relación con la prensa y las áreas de comunicaciones y vinculación de las mismas universidades. En ese sentido, resulta interesante observar resultados de estudios internacionales que evidencian esta tensión en la relación entre los investigadores y su percepción de la 
divulgación pública, y en especial mediada de la ciencia, la que temen podría ser mal interpretada o utilizada por el público (Davies 2008), o exagerada si no se trabaja periodísticamente de manera responsable (Junter 2016). Además, y asumiendo que los científicos no reciben formación para comunicar sus resultados fuera de los círculos académicos (Jucan y Jucan 2014) y por lo tanto se reconocen con escasas habilidades comunicativas (Gething 2003), se abre también la posibilidad de un poco explorado nicho de trabajo para profesionales del área de la comunicación, que pueden servir de guías, asesores e impulsores de estas capacitaciones tanto a estudiantes como a docentes en dos grandes áreas: la correcta realización de papers académicos a través de técnicas de escritura científica y coaching de presentación oral para congresos especializados, y/o como profesores en capacitaciones y talleres de escritura, expresión oral, y estrategias de comunicación de proyectos.

\section{CONCLUSIONES}

A partir de entrevistas realizadas a una muestra de investigadores, constatamos la disparidad entre la difusión y la divulgación en el trabajo académico: mientras la difusión es parte de las exigencias e indicadores laborales, la divulgación se enmarca en motivaciones de responsabilidad social de carácter personal, pero sin reconocimiento efectivo en el currículo profesional de quienes la realizan. Para equilibrar esta disparidad en la valoración de ambos espacios de comunicación de la ciencia, los académicos destacan la necesidad de reformular la política institucional universitaria, de modo que los incentivos económicos y de jerarquía académica, no se acoten a una arista de la difusión, como es la publicación de artículos científicos, sino también consideren las actividades de vinculación con el medio, paso necesario, incluso, para la coherencia de muchas universidades que declaran esta dimensión como parte fundamental de sus misiones institucionales.

Otra conclusión importante que se puede extraer de las entrevistas, y que se torna importante como desafío para periodistas, sobre todo, de instituciones de educación superior, es que, así como la sociedad tiene una imagen caricaturizada de los científicos, existe una apreciación de parte de ellos, donde se ve al periodista o a la prensa en general como profesionales que no saben tomar el peso necesario a la investigación científica, lo que genera un distanciamiento en cuanto a la poca especialización. Se desprende entonces, lo importante que son los profesionales de la comunicación para el desarrollo de la cultura científica, pero no sólo desde las tareas periodísticas tradicionales, sino también en la colaboración y formación de futuros divulgadores.

\section{REFERENCIAS}

Brownell, S. E., Price, J. V. y Steinman, L. Science communication to the general public: why we need to teach undergraduate and graduate students this skill as part of their formal scientific training. The Journal of Undergraduate Neuroscience Education, 12 (1), E6-E10 (2013)

Büchi, M. Microblogging as an extension of science reporting. doi: 10.1177/0963662516657794, Public Underst Sci., Vol 26(8), 953-968 (2017)

Clarkson, M. D., Houghton, J., Chen, C. y Rohde, J. Speaking about science: a student-led training program improves graduate students' skills in public communication, doi.org/10.22323/2.17020205, Journal of Science Communication JCOM, 17 (02), A05 (2018)

CONICYT. Informe del Consejo de CONICYT 2015-2018 Comisión Nacional de Investigación Científica y Tecnológica, Santiago, Chile (2018)

CONICYT. Encuesta Nacional de Percepción Social de la Ciencia y la Tecnología en Chile. Comisión Nacional de Investigación Científica y Tecnológica, Santiago, Chile (2016)

Crue y FECYT. Guía de Valoración de la Actividad de Divulgación Científica del Personal Académico e Investigador. Crue Universidades Españolas y la Fundación Española para la Ciencia y la Tecnología (FECYT), Madrid, España (2018)

Davies, S. Constructing Communication. Talking to Scientists About Talking to the Public, doi: 10.1177/1075547008316222, Science Communication, Vol. 29 (4), 413-434 (2008)

Espinosa, V. Difusión y divulgación de la investigación científica, doi: 10.4067/S0718-34292010000300001, IDESIA, Vol. 28, 5-6 (2010)

Farinella, M. The potential of comics in science communication, doi.org/10.22323/2.17010401, Journal of Science Communication JCOM 17 (01), Y01 (2018)

Gething, L. 'Them and us': Scientists and the media - attitudes and experiences, SAMJ, Vol 93 (08), 197-201 (2003)

Glaser, B. y Strauss, A. The Discovery of Grounded Theory: strategies for qualitative research. 1er ed., Routledge, Nueva York, Estados Unidos (1967)

Guenther, L. y Joubert, M. Science communication as a filed of research: identifying trends, challenges and gaps by analyzing research papers. Journal of Science Communication JCOM, 16 (2), A02 (2017) 
Guzmán, C. El disfraz de la competitividad y la producción de conocimientos en el capitalismo cognitivo, ISSN 2256-5485, Revista Colombiana de Sociología, Vol.32(1), 43-60 (2009)

Hunter, P. The communications gap between scientists and public. doi: 10.15252/embr.201643379, EMBO reports, Vol 17 (11), 1513-1515 (2016)

Jucan, M. y Jucan C. The Power of Science Communication, Procedia - Social and Behavioral Sciences, 149, 461-466 (2014)

Kahan, D. What is the 'science of science communication'? Journal of Science Communication JCOM, 14 (3), Y04 (2015)

Lamberts, R. Science communication: recently public, occasionally intellectual. Journal of Science Communication JCOM, 16 (1), C01 (2017)

Lascurain, M. y Sanz, E. La divulgación científica en el entorno universitario, en Borges, M. (coord.) A ciencia da informaçao criadora do conhecimiento, Vol 1, 507-516. Universidad de Coimbra, Portugal (2009)

Mato, D. Contribución de experiencias de vinculación social de las universidades al mejoramiento de la calidad académica y factores que limitan su desarrollo y valoración institucional. Avaliaçao: Revista de Avaliaçao da Educaçao Superior, Vol. $18(1), 151-180(2013)$

Miller, S. y Fahy, D. Can Science Communication Workshops Train Scientists for Reflexive Public Engagement?. Science Communication, 31 (1), 116-126 (2009)

Ministerio de Economía. VII Encuesta Nacional sobre Gasto y Personal en Investigación y Desarrollo (año de referencia 2016) Ministerio de Economía, Fomento y Turismo, Santiago, Chile (2018)

Ministerio de Economía. VI Encuesta Nacional sobre Gasto y Personal en Investigación y Desarrollo (año de referencia 2015) Ministerio de Economía, Fomento y Turismo, Santiago, Chile (2017)

Montenegro Martínez, M. y Pujol Tarrés, J. La fábrica de conocimientos: in/corporación del capitalismo cognitivo en el contexto universitario, doi: 10.5565/rev/athenead/v13n1.1031, Athenea Digital. Revista de pensamiento e investigación social, 13(1), 139-154 (2013)

Perez, LL. y Olvera, MD. Public communication of science in Spain: a history yet to be written. Journal of Science Communication JCOM, 16 (3), Y02 (2017)

Silva, J. y Bultitude, K. Best practice in communications training for public engagement with science, technology, engineering and mathematics, doi.org/10.22323/2.08020203, Journal of Science Communication JCOM, 8(2), A03 (2009)

Stuart, A. E. Engaging the audience: developing presentation skills in science students. Journal of Undergraduate Neuroscience Education, Vol. 12 (1), A4-A10 (2013) 
